Cahiers de recherches médiévales

Tristan et Iseut chez les Celtes ou la tentation de la pureté dans quelques adaptations tristaniennes post-bédieristes

Alain Corbellari

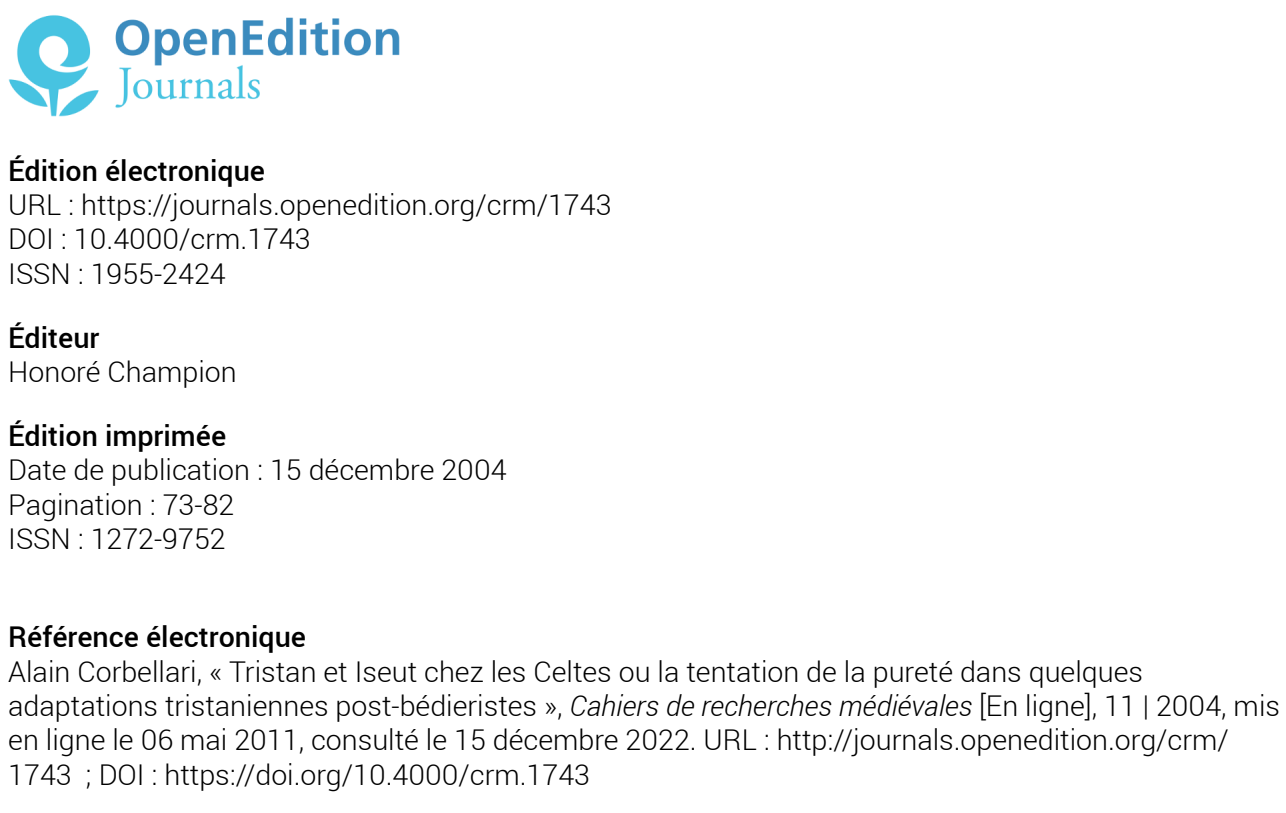

Ce document a été généré automatiquement le 15 décembre 2022.

Tous droits réservés 


\title{
Tristan et Iseut chez les Celtes ou la tentation de la pureté dans quelques adaptations tristaniennes post- bédieristes
}

\author{
Alain Corbellari
}

1 On aurait pu croire que la perfection unanimement saluée du Roman de Tristan et Iseut renouvelé par Joseph Bédier (1900) découragerait les adaptateurs de tenter d'autres reconstitutions de cette fameuse « histoire d'amour et de mort » : ce fut le contraire qui se produisit, et il n'existe pas aujourd'hui de légende médiévale qui ait suscité, et de très loin, autant de réécritures que celle des amants de Cornouailles. Aux vingt-deux renouvellements français postérieurs à Bédier que je citais dans ma thèse ${ }^{1}$, et après une accalmie d'une dizaine d'années ${ }^{2}$, sont venues presque coup sur coup s'ajouter, à exactement cent ans du renouvellement bédiériste, deux adaptations d'une originalité certaine : La Folie du roi Marc de Clara Dupont-Monod ${ }^{3}$ et le Iseult et Tristan de Yann Brekilien 4 .

2 Un seul point commun, souligné dans les deux cas par le titre, rapproche ces romans: leur volonté de focaliser le récit sur un personnage habituellement moins valorisé de la légende. Mais, au delà de cette revendication d'originalité, tout sépare ces deux textes, dans les moyens utilisés comme dans les buts recherchés. L'ambition du premier est d'ordre avant tout romanesque: si la trame popularisée par Bédier y est bien reconnaissable, elle y est diffractée et redistribuée en un enchevêtrement temporel complexe. Long monologue torturé du roi trompé, il n'évite pas toujours les lourdeurs et les contorsions lorsqu'il s'agit de faire raconter par ce narrateur trop impliqué des moments de la vie des amants qui lui restent traditionnellement inconnus. Pour Clara Dupont-Monod, le roi Marc n'est dupe qu'en apparence du jeu du couple adultère : à la fois masochiste et victime du devoir de dignité incombant à sa charge, il souffre en silence, retirant un sombre orgueil d'un savoir qui lui fait toujours prévoir d'avance les 
ruses des amants, mais qui doit rester caché sous peine de voir anéantie la (fragile et, en fin de compte, illusoire) supériorité qu'il lui donne.

3 La phrase d'ouverture, dans sa crudité elliptique très « fin de $\left(\mathrm{XX}^{\mathrm{e}}\right)$ siècle » annonce la couleur :

Je m'appelle Marc, je suis roi de Cornouailles et ma femme me trompe. ${ }^{5}$

4 Et cette position «non-dupe » du roi n'est pas sans suggérer une relecture parfois audacieuse des textes médiévaux. Ainsi la scène, si étrange, de la surprise des amants par Marc dans la forêt du Morois, chez Béroul, en reçoit-elle un éclairage non dénué d'intérêt :

Je décide de la croire fidèle. Je décide, là, maintenant, devant Tristan et Yseut endormis, qu'un corps vêtu d'une chemise, qu'une épée séparant deux corps, qu'un sommeil aussi tranquille, garantissent la chasteté. Je le décide; reste à m'en convaincre. ${ }^{6}$

5 Ailleurs, tel trait de mœurs étonne; ainsi le bref épisode du peintre venu «faire le portrait » de Marc (ce dernier le trouve même « très ressemblant ") apparait-il comme un anachronisme peu utile dans la trame du récit, et l'écriture n'échappe pas toujours au didactisme. On se posera par exemple la question de la pertinence de cette notation qui s'insère un peu en porte-à-faux (le détail mythique devient commentaire pour guide Michelin) dans le monologue du roi au moment où, juché dans le pin, il entend venir les amants :

J'ai froid. J'entends un bruit. On raconte que Tintagel a été construit par des géants, et qu'il devient invisible deux fois par an, une fois en été, l'autre en hiver. ${ }^{8}$

6 En bref, donc, sans être une réussite totale, ce roman marqué par une certaine préciosité et qui fut honorablement accueilli lors de la rentrée littéraire 2000, constitue sans aucun doute l'une des versions modernes les plus travaillées et les plus purement littéraires de la légende.

7 Le texte de Yann Brekilien, polygraphe bien connu des milieux celtomanes, avoue, au contraire, d'entrée de jeu qu'il sert une cause : celle de la culture celte brimée, étouffée, etc. par des envahisseurs ingrats qui, pourtant, lui doivent tout. De Renan et Hersart de la Villemarqué aux plus modernes revendications autonomistes, on connait cette vieille rengaine misérabiliste qui a toujours été accompagnée d'une volonté de récrire quelques-uns des plus grands textes de la littérature médiévale. Il ne s'agit pas ici de refaire l'historique de cette vaste querelle ${ }^{9}$; plus modestement, on s'attachera, afin de mieux dégager l'originalité (réelle) du propos de Brekilien, à en repérer les traces à travers les adaptations post-bédiéristes de la matière tristanienne, terrain de polémiques privilégié dans la mesure où près de la moitié de ces réécritures s'attache plus ou moins explicitement à réévaluer à la hausse l'importance du substrat celtique de la légende.

Certes, on ne s'étonnera pas de trouver l'explication de cette velléité récurrente dans certain caractère, à la fois réel et fantasmé, volontiers prêté à l'adaptation de Bédier, savant dont la personnalité semblait attirer les controverses et dont il convient de rappeler brièvement les traits les plus saillants ${ }^{10}$. On sait en effet que, bien qu'ayant passé presque toute son enfance dans l'île de la Réunion, Joseph Bédier, qui pouvait de surcroît s'enorgueillir de racines normandes, voire bretonnes, ne se signala guère, dans l'Université française comme un partisan de la «France des régions »: son parcours (études puis enseignement à l'École Normale Supérieure et au Collège de France, élection à l'Académie française, cursus complet de la Légion d'honneur, multiples et 
variées missions politiques et administratives allant du déchiffrage des carnets de soldats allemands lors de la Grande Guerre à la présidence de l'Alliance française, en passant par de nombreux voyages protocolaires à l'étranger) est un modèle de carrière officielle dans la "Troisième République des lettres" et ses idées scientifiques ellesmêmes n'ont pas peu contribué à l'instauration d'une vulgate jacobine de l'histoire littéraire française: dans Les Fabliaux comme dans Les Légendes épiques, il défend l'origine autochtone des genres qu'il étudie et dans son édition du Tristan de Thomas il minimise l'importance de l'apport celte dans la constitution de la légende, poursuivant, sur la lancée des considérations de l'Allemand (!) Wendelin Foerster qui avait soutenu, deux décennies auparavant, que Chrétien de Troyes avait lui-même inventé l'essentiel de sa thématique, la revendication pour la France seule des innovations de la « matière de Bretagne ». En 1924, la revue bretonne Breiz Atao s'en prendra même vertement à Bédier, l'accusant de ne pas avoir rendu justice, dans sa grande Histoire illustrée de la littérature française, parue chez Larousse (les pages incriminées sont en fait de Faral, mais Bédier était directeur de la publication et l'on ne prête qu'aux riches), à l'inspiration bretonne des récits de la Table Ronde ${ }^{11}$.

Bédier se défendra peu de ces attaques, mais la question reste entière de savoir si réellement son Roman de Tristan et Iseut reflète autant que certains ont pu le sousentendre, une vision farouchement « centralisatrice » de la matière de Bretagne.

Donnant en 1972 au «Livre de Poche » une version censée remplacer celle d'André Mary, passée dans la collection concurrente («Folio ») suite au retrait de Gallimard du groupe Hachette, René Louis, universitaire comme Bédier, justifiait ses divergences d'avec ce dernier (sans une seule fois évoquer l'adaptation de Mary), en prétendant donner "une image plus fidèle $[\mathrm{du}]$ conte primitif, tout imprégné de traditions celtiques $»^{12}$; avouant par ailleurs : «il n'est pas un seul épisode où je n'aie fait miennes quelques-unes des heureuses trouvailles de style qui font le prix et le charme impérissable du renouvellement de Joseph Bédier", Louis n'a infléchi la trame bédiériste qu'en des points de détails, le plus important étant, comme il l'indique luimême ${ }^{13}$, celui de la signification du philtre. Et cette discussion, qui occupe une quinzaine de pages des « Notes et commentaires » de Louis, intéresse autant sinon plus la question de la reconstitution du «roman primitif » que celle de l'évaluation des éléments celtiques.

11 Si les versions de Michel Manoll, pour qui « il ne fait aucun doute que le poème de Tristan et Iseut soit d'origine celtique ${ }^{14}$, et de Michel Cazenave, qui s'honore d'une préface signée par Jean Markale ${ }^{15}$, se veulent ouvertement celtisantes et omettent toute référence explicite à Bédier (alors qu'elles pillent sans vergogne sa trame narrative), le Tristan et Iseut de Pierre d'Espezel comprend en revanche une préface de Jean Marx qui, en tant qu'universitaire, ne peut faire l'économie de la polémique avec le grand ancien. Les termes en sont intéressants :

Joseph Bédier, qui, avec un si merveilleux talent, s'est employé à restituer ce qu'il pensait être le premier état du poème, $\mathrm{y}$ a vu un thème essentiel: la lutte entre l'amour et l'honneur, entre l'amour et la loi sociale, une sorte de roman tragique de l'adultère. En notre temps, nous sommes tentés, sans oublier cet aspect, de retrouver dans la légende, des traits et un ensemble beaucoup plus rudes, plus barbares, plus proches à la fois des antécédents celtiques et d'une atmosphère féodale moins raffinée. C'est en ce sens que $M$. d'Espezel a réuni et traduit avec grand art les textes authentiques qui composent le présent livre; ils donnent un éclairage à la légende qui, pour être nouveau, n'en est ni moins saisissant ni moins beau. ${ }^{16}$ 

recherche du "premier état du poème " par Bédier, Marx ne fait pas allusion à son Roman de Tristan et Iseut, qui n'a guère cette ambition, mais au travail scientifique inclus dans l'édition du Tristan de Thomas; pourtant, il le met en parallèle avec la réécriture romanesque de D’Espezel. Par ailleurs, quels sont ces «textes authentiques » évoqués par Marx ? Les récits celtiques que Gertrude Schoepperle, entre autres, a ramenés au jour, les présentant comme les antécédents probables de la légende tristanienne (Diarmaid et Grainne, etc.) ${ }^{17}$, en sont extrêmement éloignés, et les utiliser reviendrait à écrire une toute autre histoire. En réalité, D'Espezel s'est servi des mêmes sources que Bédier, à savoir d'abord les textes français et en second lieu leurs dérivés germaniques. La langue même de son adaptation, toute pleine d'archaïsmes modérés, s'autorise de celle de Bédier ${ }^{18}$. D'ailleurs, Louis, Cazenave et Manoll, s'ils usent d'un français plus contemporain (disons plus "neutre»), n'en articulent pas moins identiquement leur reconstitution sur une structure narrative directement dérivée de celle de Bédier; Louis a beau truffer son texte de détails inspirés par l'histoire de la civilisation médiévale ${ }^{19}$ et Cazenave ajouter lyriquement des résurgences du topos printanier ${ }^{20}$, on chercherait en vain la marque certaine, dans ces divers textes, d'une réelle « celtisation».

L'entreprise de Yann Brekilien s'avère, dans ce contexte, d'une tout autre envergure : elle est en effet la première à faire éclater complètement la trame bédiériste que même les adaptateurs les plus libres et les plus récents, Catherine Hermary-Vieille ${ }^{21}$ (en dépit de ses nombreux emprunts au roman en prose), Pierre Dalle Nogare ${ }^{22}$ (en dépit de son caractère extrêmement elliptique) ou Clara Dupont-Monod (en dépit des changements de perspective que nous signalions plus haut) respectaient encore. dans la préface, que les textes de Béroul et de Gottfried de Strasbourg sont de "pâles imitations » des originaux celtiques prouve au moins deux choses : l'adjectif «pâles » démontre qu'il ne les a pas lus et le substantif «imitation" qu'il se fait une idée parfaitement naïve du concept d'influence et qu'il n'a aucune idée de ce que subsume l'idée même de l'écriture. Cinquante ans avant Kristeva et Derrida, Bédier avait lumineusement montré - et que ce plaidoyer ait pu avoir un caractère pro domo n'ôte rien à sa pertinence - que toute réécriture effaçait toujours ses antécédents au point de rendre sans objet toute recherche d'origine ${ }^{23}$. De surcroit, le texte que "restitue " Brekilien est si éloigné de la légende dont on croit avoir l'habitude qu'il ne peut y avoir que de la mauvaise foi à dénier toute originalité aux textes soi-disant "postérieurs " aux prototypes celtiques dont il dit s'inspirer. Quant à créer de toutes pièces un « original » fictif (un «hypertexte hypotextifié » disait Genette ${ }^{24}$ ), Bédier avait su le faire avec autrement plus d'autorité !

serait injuste de juger tout ce travail de réécriture sur deux mots malheureux, car travail il y a incontestablement eu, et d'une radicalité qui, comme nous le disions plus haut, balaie sans rémission toutes les tentatives précédentes, en fait étonnamment timorées, de retrouver un «Tristan celtique ».

Le récit commence sur les remparts de la forteresse de Marc, qu'arpente nerveusement Iseult, promise sans son consentement au «chef du clan du cheval »: Brekilien insiste lourdement ici sur le sens celte du nom de Marc'h et en profite pour nous conter, première d'une série de digressions qui apparentent son texte au roman à tiroirs, la légende du roi aux oreilles de cheval (p.17-25), apparemment empruntée au corpus

Cahiers de recherches médiévales, 11 | 2004 
réuni par Gaël Milin ${ }^{25}$. La technique du récit rapporté sera utilisée à d'autres reprises dans le cours du texte; ainsi nous seront rappelées les légendes de la ville d'Ys (p. 18-19) et de Merlin (p. 125-27). On aura aussi reconnu au passage l'histoire d'Yvain, redevenu Owein (p. 40), la mention des fameuses triades celtiques (p.51), une allusion à l'Eliduc de Marie de France (p.128) et même des relents de l'histoire de Barbe-Bleue (p. 27). Quant à Caradoc, qui était un soupirant d'Iseut dans le roman de Thomas, il devient (ironie voulue ?) le cheval de Tristan! (p. 47)

On n'insistera pas sur la bretonnisation de presque tous les noms propres : mais à ce taux-là pourquoi ne pas être allé jusqu'au bout et ne pas avoir parlé de Drystan et Essylt ${ }^{26}$ ? D'autres velléités de l'auteur semblent également davantage des vœux pieux que des principes rigoureux de composition. Ainsi rien ne vient, dans le texte, nous rappeler formellement la volonté bien affirmée de l'auteur de faire vivre ses personnages "aux temps antiques" et de les voir mener "une existence simple et proche de la nature " (introduction, p. 11) ${ }^{27}$. Au contraire, la présence d'un puissant "château-fort» (p. 27) nous ramène au moins à ce XII ${ }^{\mathrm{e}}$ siècle que Brekilien disait fuir. On lui rappellera par la même occasion que les «échecs » (p. 108) étaient inconnus en Occident avant le $\mathrm{XI}^{\mathrm{e}}$ siècle (malgré la fortune d'un soi-disant « jeu de Charlemagne ») et que les «olifants» (p.33) font bien peu celtiques. En évoquant le «biniou » et la «bombarde» (p. 23), on retrouve peut-être la «Bretagne éternelle », mais on s'éloigne encore davantage de l'Antiquité. Au titre des détails, l'intrusion d'une "chlamyde " (p. 46: mot grec) et d'une "roque» (id.: mot germanique!) détonne et l'on se permettra de ne pas être convaincu par l'opportunité de termes tels «salon de coiffure » (p. 20), « secret d'État» (id.) ou « opinion publique » (p. 21).

L'insistance nationaliste sur «nos conceptions celtiques » (p. 30) lasse vite, quand il ne s'agit pas de polémiques plus obscures encore :

Aucun pédant, en effet, n'avait eu l'idée d'enseigner qu'il avait existé très anciennement une " préhistoire » tronçonnée, comme un saucisson, en tranches d'épaisseur inégale qu'il était de bon ton, pour paraître instruit, d'appeler 《 paléolithique 》, 《 mésolithique », « néolithique 》, « chalcolithique 》 et « âge des métaux 》 (p. 51).

Le moins que l'on puisse dire d'une telle notation est qu'on en voit mal la pertinence (et qui est le pédant ici?), sinon à sous-entendre que tel âge "celte » de La Tène ou de Hallstatt ne se laisse pas réduire à un tel « saucissonnage » érudit, ce qui est tout de même une certaine preuve d'ingratitude envers des savants qui ont su revaloriser le très ancien passé celtique de l'Europe !

Outre les ruptures de rythmes que le style démonstratif de Brekilien multiplie, il convient de se demander si la dévalorisation que l'auteur fait subir au personnage de Tristan est réellement la meilleure façon de rendre hommage à la féminité et au courage de son héroïne. Il ne manque en effet pas de commentateurs pour faire remarquer que dans le Tristan de Béroul c'est déjà Iseut qui semble, en plus d'un épisode, imposer ses vues à Tristan bien plus qu'elle n'est sa compagne soumise ${ }^{28}$ : la question se pose donc de savoir s'il était nécessaire d'aller chercher plus loin un personnage de «femme celte » alliant à la perfection courage et sensualité, intelligence et instinct. Toujours est-il que Brekilien se sent obligé de renchérir et fait de son Iseult une sorte de virago cynique proférant des rodomontades où se fait jour un esprit qu'il faut bien qualifier de raciste :

-Je suis fille de Bretagne, je suis donc une Celte et tout Celte, contrairement aux Latins, aux Germains et aux peuples d'Orient, est un être libre (p. 25). 
voit ainsi provoquer indiscrètement Tristan sur sa virilité, dont elle a d'ailleurs "grand hâte» (p.54) de pouvoir juger, et se félicite finalement de son amant comme une vieille coquette de son gigolo:

Quelle chance, pensait-elle, que ce soit à mon Tristan que j'ai eu naguère l'heureuse inspiration de jeter mon geis de danger et de destruction pour qu'il m'enlève et m'emporte loin du château de Tintagel et du vieux roi qui me convoitait! (p. 164) La niaiserie de Tristan ne fait d'ailleurs aucun doute, jugée du moins à l'aune des répliques très fleur bleue que lui prête Brekilien :

Il lui embrassa dévotement la main et déclara:

-Tu n'es pas reine du pays de Cornouailles, c'est entendu. Mais tu es reine de mon cœur et c'est cela qui importe (p. 57).

Il est vrai que, sources celtiques obligent, il ne pouvait plus réellement être question d'amour entre Tristan et Iseult ; ainsi voit-on Brekilien adapter dans un premier temps assez fidèlement la légende de Diarmaid et Grainné : le philtre redevient geis, et la fuite, la vie dans la forêt et l'épisode de l'eau aventureuse se trouvent rapportés aux seuls Tristan et Iseult. Ce faisant, l'auteur est amené à supprimer tous les épisodes liés, au sens large, aux «enfances" de Tristan, puisque montrer le courage de ce dernier ternirait l'aura de son amante. Doit-on pourtant rappeler l'évidence de l'intertexte celtique dans les récits de lutte contre le Morholt ou le dragon? Ainsi ce roman " celte » se sent-il obligé de trier parmi ses sources et de se priver de précieux appoints à la thèse qu'il défend. En même temps, Brekilien reste malgré tout soucieux de restituer le plus de matière possible à ses soi-disant sources bretonnes et on peut comprendre qu'il ne résiste pas à l'évocation massive des chevaliers du roi Arthur ; ce faisant, il renoue (sans doute involontairement) avec une innovation des prosateurs français du XIII ${ }^{\mathrm{e}}$ siècle ! Il est vrai qu'intégrer Tristan à la confrérie de la Table Ronde permet de nous apprendre que le nom de Myrddin a été transformé par «les descendants des Gaulois [...] en Merlin, ce qui ne veut rien dire, mais est plus facile à prononcer " (p. 125) ${ }^{29}$, de rattacher Arthur au "clan de l'Ours", puisque «son nom venait du mot celtique Arth désignant le sympathique [sic !] plantigrade » (p. 135) et de donner à Excalibur son nom celte de Kaledvoulc'h, " dont personne n'avait encore eu le mauvais goût de déformer le nom en Excalibur, ce qui ne veut rien dire dans aucune langue » (p. 145). On rappellera tout de même, quant à ce dernier point, que l'essentiel de la geste d'Arthur nous est connu par les textes latins de Geoffroy de Monmouth et qu'il a fallu aller rechercher des parallèles jusque chez les Ossètes pour expliquer de manière convaincante la scène finale du roman français de La Mort le Roi Artu ${ }^{30}$. De tout cela, Brekilien, tout à sa celtomanie, semble n'avoir cure.

Originalité certaine de sa version, nous sommes encore loin de la fin de cet Iseult et Tristan lorsque intervient le grand effondrement du monde arthurien, ce qui tranche sur toute tradition, l'histoire des amants de Cornouailles étant traditionnellement incluse à l'intérieur du "grand temps» de la geste arthurienne ${ }^{31}$ : Brekilien est donc obligé de trouver une façon inédite de conclure son récit. C'est la présence de Morgane, venue reprendre le corps des héros après la bataille de Salesbières, qui lui donne l'idée de récupérer le thème de la voile noire, avec une obstination qui, d'ailleurs, peut paraître étrange, puisque ce motif entretient, comme on le sait, des rapports autant sinon plus nets avec le monde gréco-romain qu'avec l'univers celtique. En fait, on comprend vite que Brekilien a trouvé une fois de plus un moyen de prêcher pour sa paroisse celto-féministe. En l'absence de toute seconde Iseut, c'est Morgane qui hérite 
de ses prérogatives. Blessé après la bataille, Tristan est donc soigné en Avallon et attend le retour d'Iseut, qu'une voile blanche doit annoncer. Morgane prétend voir une voile noire sur la mer, Tristan s'inquiète, mais, heureux coup de théâtre, la méprise n'était pas volontaire et les justifications du narrateur et de son personnage transforment cette scène tragique en un contretemps presque comique, d'un effet qui n'est pas très éloigné du vaudeville :

Bien sûr, il est arrivé dans l'Histoire qu'une femme annonçât une voile noire pour tromper l'homme qui espérait une voile blanche, mais elle le faisait par jalousie amoureuse et Morgane n'avait aucune raison d'être jalouse d'Iseult. Il ne lui paraissait pas imaginable qu'elle pût être amoureuse de lui, malgré les touchantes attentions dont elle l'avait couvert quand elle lui servait de garde-malade et les baisers furtifs qu'il lui était arrivé de poser sur son front quand elle croyait qu'il ne s'en rendrait pas compte.

-Eh bien, elle n'est ni blanche ni noire, fit-elle d'un ton triomphant, à son retour dans la chambre. C'est une belle voile solide de grosse toile rouge sombre. Mais à contre-jour et dans l'ombre du petit matin elle paraissait encore plus sombre et c'est pourquoi je l'ai dite noire. Je m'excuse de cette erreur (p. 154-55).

On laissera à chacun le soin de juger si la «femme celte» sort grandie de cette présentation en infirmière aussi dévouée que maladroite.

Iseut retrouve ainsi Tristan sain et sauf et, désormais, les amants vivront dans une sorte de hors-temps idyllique, manière de paradis retrouvé, où ils pourront en toute quiétude cueillir des champignons et savourer des myrtilles (p. 163), quand ils ne chanteront pas le lai du chèvrefeuille composé par Tristan (p. 164). Et comme il faut bien terminer l'histoire, Brekilien retrouve en conclusion le motif du sanglier blessant à mort Tristan, et imagine que le roi Marc'h, qui accompagnait le héros, montre si peu de zèle pour lui apporter de l'eau qu'il le laisse mourir de sa blessure. Gourdival « jeta, en conséquence, sur lui sa malédiction pour l'éternité » (p. 172), ce qui ne semble que peu émouvoir le souverain. Le récit s'achève sur le motif de la ronce qui pousse sur le tombeau des amants, motif dont l'aspect celtique, une fois de plus, demeure problématique.

On aura compris que Brekilien reste très loin de nous avoir restitué un véritable Tristan celte qui pût nous faire oublier les versions modernes canoniques. Il y a en fait ici confusion sur les termes; le texte de Brekilien est, comme on l'a vu, un patchwork qui doit bien plus d'éléments qu'il ne veut l'avouer aux textes français, allemands et latins (!) des $\mathrm{XII}^{\mathrm{e}}$ et XIII ${ }^{\mathrm{e}}$ siècles. Au surplus, il n'est même pas sûr qu'un lecteur un peu superficiel ferait d'emblée le lien avec la légende tristanienne si l'on s'avisait de modifier les noms des personnages principaux. En dernière analyse, on revient toujours au principe simple que la conviction ne saurait s'emporter qu'à hauteur du talent manifesté dans le nouveau texte. Or, à la fois trop démonstratif de son idéologie et d'une mise en œuvre romanesque trop indécise, cet Iseult et Tristan nous étonnerait beaucoup s'il parvenait à s'imposer comme le classique qu'il a l'ambition de devenir...

Il nous faut conclure en revenant sur la question, que nous avions laissée pendante, de ce qui, dans l'adaptation de Bédier, a pu pareillement déchaîner les foudres des amis de la Bretagne. Pour ce faire, rien ne paraît plus parlant que d'évoquer un texte qui a parfois été présenté à tort comme une version autonome de la légende de Tristan: le texte breton, déjà ancien, de Xavier de Langlais ${ }^{32}$. Contrairement, en effet, à ce qu'ont pu croire certains celtophiles, ce n'est pas un texte original, mais une pure et simple traduction de l'adaptation de Bédier : on y retrouve jusqu'à cette pudeur victorienne qui dénature et obscurcit la teneur des serments ambigus (Iseut parle de «celui qui, le 
premier, [l']a prise vierge entre ses bras » et des hommes qui l'ont « tenue entre [leurs] bras »), et si le nombre des chapitres est différent, c'est uniquement parce que Langlais a très légèrement augmenté l'épilogue, au point d'en faire un vingtième chapitre séparé. À peine d'ailleurs peut-on distinguer, çà et là, quelques lignes qui sont davantage des libertés que s'est accordé le traducteur par rapport à son texte source que de réelles innovations, et les quelques variantes de noms propres ("Olwenn» transposant heureusement le nom trop français de Blanchefleur, Iseut aux Blanches Mains devenant «Iseut au sourire d'ange » et l'« Ankou » personnifiant le trépas dans la plus pure tradition bretonne, là où Bédier évoquait simplement «la mort») ne représentent guère que de menus détails.

Que plus d'un celtisant s'y soit trompé ${ }^{33}$ en dit cependant long: comment, a priori, imaginer que l'on puisse confondre la version (prétendument jacobine) de Bédier avec un texte qui revendique hautement sa bretonnité ? Faut-il croire que Bédier avait su faire parler plus fort qu'il ne le croyait ses propres (mais bien lointaines) racines armoricaines? Mieux vaudrait sans doute y voir la preuve non seulement que le procès intenté à Bédier infère abusivement de ses positions critiques à sa pratique romanesque et cela sans doute parce que, n'en déplaise aux nationalistes de tout poil, la réussite artistique semble bien, en vertu d'une loi qui est la condition même de toute écriture littéraire consciente d'elle-même, repousser au second plan les spécificités ethniques et idéologiques qu'elle pourrait véhiculer. Il est donc vain de chercher des couleurs particulières là où une certaine qualité d'inspiration a réussi à hausser un récit particulier à un degré d'universalité qui rend superflu tout rappel trop précis de ses origines. Ne retrouvons-nous pas là, en fin de compte, l'une des définitions possibles du mythe?

\section{NOTES}

1. Joseph Bédier écrivain et philologue, Genève, Droz, «Publications romanes et françaises ", CCXX, 1997, pp. 735-36. Notons que je recensais aussi quatre adaptations anglaises, auxquelles il faudrait encore ajouter le Brazil et la nouvelle «Tristan and Iseult » de John Updike, transpositions très libres que, pour cette raison, je cite ailleurs dans ma bibliographie (p. 740) et pour l'analyse desquelles je renvoie à mon article "John Updike's Tristanian Passion», Tristania, 19, 1999, pp. 115-26. En élargissant le concept d'« adaptation ", on pourrait d'ailleurs être amené à considérer comme dérivés du Roman de Tristan et Iseut.Un Jardin sur l'Oronte de Barrès, le Sparkenbroke de Charles Morgan, Les Enfants du roi Marc de Roger-Louis Junod, voire La Rose des sables de Montherlant, mais la liste menacerait ainsi de devenir infinie.

2. Si l'on met entre parenthèses la curieuse tentative d'André Miquel (Tristan et Iseut d'après Joseph Bédier, Paris, Odile Jacob, 1996) qui est une pure et simple mise en alexandrins du roman de Bédier, le dernier renouvellement créateur était Le Rivage des adieux de Catherine Hermary-Vieille, Paris, Pygmalion/Gérard Wathelet, 1990. Sur cette adaptation et sur quelques autres, je renvoie à mes articles « Joseph Bédier : (d') écrire la passion. L'écriture et la fortune du Roman de Tristan et Iseut ", in Michèle Gally (éd.), 
La Trace médiévale et les écrivains d'aujourd'hui, Paris, PUF, "Perspectives littéraires ", 2000, pp.107-20 ; «Le Rendez-vous épié de Tristan et Iseut», in Walter Lenschen (Hrsg.), Die Sprachen der Liebe / Langages de l'amour, Bern, Lang, 2000, pp. 219-32 et «Tristan et Iseut sous le pin : Béroul vu par trois adaptateurs », in L'amour des lettres. Festschrift für Walter Lenschen, éd. par A. Schwarz et A. Vacek, Bern, Lang, 1999, pp. 37-48.

3. Clara Dupont-Monod, La Folie du roi Marc, Paris, Grasset, 2000. Je remercie M. Jacques Chessex de m'avoir rendu attentif à ce roman.

4. Yann Brekilien, Iseult et Tristan, Monaco, Le Rocher, 2001.

5. La Folie du roi Marc, p. 11.

6. Ibid., p. 130.

7. Ibid., p. 211.

8. Ibid., p. 76.

9. Voir notamment Michel Zink, Le Moyen Âge et ses chansons, Paris, De Fallois, 1995.

10. Pour plus de détails, voir Joseph Bédier écrivain et philologue, op. cit., passim.

11. Sur cet épisode, voir Joseph Bédier écrivain et philologue, op. cit., p. 453-54.

12. René Louis, Tristan et lseut, renouvelé en français moderne, Paris, Librairie Générale Française, coll. « Le Livre de Poche », 1972, p. 301.

13. Ibid., p. 286.

14. Michel Manoll, Tristan et Yseult, Paris, G. P., 1959, 1971 ; version définitive : Paris, Jean Picollec, 1980, p. 10.

15. Michel Cazenave, Tristan et Iseut, Paris, Albin Michel, 1985. Markale n'a d'ailleurs pas d'autre source à proposer à la légende que « les Béroul, les Thomas d'Angleterre, les Eilhart d'Oberg, les Gottfried de Strasbourg » (p. 3).

16. Pierre d'Espezel, Tristan et Iseut, Paris, Union latine d'Éditions, 1956, p. v.

17. Gertrude Schoepperle, Tristan and Isolt, a study of the sources of the romance, Frankfurt / London / New York University, Ottendorfer Series of Germanic Monographs 6 et 7, 1913.

18. J'insiste sur le rôle de modèle qu'a joué la prose de Bédier dans le domaine des traductions des textes en ancien français dans Joseph Bédier écrivain et philologue, op. cit., pp. 266-290 et aussi dans «Traduire ou ne pas traduire : le dilemme de Bédier. À propos de la traduction de la Chanson de Roland ", Vox Romanica, 56, 1997, p. 63-82.

19. Voir «Joseph Bédier: (d') écrire la passion. L'écriture et la fortune du Roman de Tristan et Iseut », art. cit., p. 118.

20. Voir ibid., pp. 113-14.

21. Op. cit.

22. Pierre Dalle Nogare, Tristan et Iseut, Paris, Philippe Lebaud, "Les intemporels », 1996, avec une préface de Michel Cazenave (1 $1^{\mathrm{e}}$ éd. 1977).

23. Voir Joseph Bédier écrivain et philologue, op. cit., à compléter avec « Relire les légendes épiques ", in L'Epopée romane au Moyen Âge et aux temps modernes, actes du XIV ${ }^{\mathrm{e}}$ Congrès international de la Société Rencesvals, Naples, 24-30 juillet 1997, publiés par Salvatore Luongo, Naples : Fridericiana Editrice Universitaria, 2001, 2 vol, t. II, pp. 843-56.

24. Gérard Genette, Palimpsestes, Seuil, « Poétique », 1982, p. 249.

25. Gaël Milin, Le Roi Marc aux oreilles de cheval, Genève, Droz, 1991. Précisons que Brekilien ne cite nommément aucune de ses sources, précaution dont nous n'allons pas tarder à comprendre toute l'utilité.

26. De ce point de vue les concepteurs de la belle série de bandes dessinées «Arthur une épopée celtique » ont été conséquents, puisqu'ils ont intitulé leur reprise du mythe tristanien Drystan et Esyllt (par Chauvel, Lereculey et Simon, Paris, Delcourt, 2002). 
Pourtant, paradoxalement, il s'agit là du moins idiomatique des albums de cette série dont l'ambition férocement archaïsante est patente, la volonté de reprise des prototypes celtiques y étant constamment battue en brèche (bien plus que chez Brekilien) par les souvenirs de la version française de la légende.

27. On peut par contre citer comme exemple réussi d'une telle volonté (certes, la dimension graphique y aide beaucoup) les albums $\mathrm{BD}$, mentionnés à la note précédente, de la série « Arthur une épopée celtique ».

28. Ce point de vue est entre autres soutenu par Barbara Nelson Sargent-Baur, "Between Fabliau and Romance : Love and Rivalry in Béroul's Tristan ", Romania, 105, 1984, pp. 292-311 ; Jacques Ribard, "Tristan/Renard revisité », Mélanges offerts à Hans Erich Keller, Kalamazoo, 1993, pp. 181-94 et E. Jane Burns, « Why Beauty Laughs : Iseut's Enormous Things ", chapitre 5 de Bodytalk: When Women Speak in Old French Romance, Philadelphie, University of Pennsylvania Press, 1993, pp. 203-40.

29. Comme si un nom propre devait signifier quelque chose! Mais l'aspect méprisant de cette notation est évident après le fastidieux excursus étymologique par lequel Brekilien nous a appris que le nom de Merlin dérivait «du mot marzh qui, dans le dialecte armoricain signifie 'merveille, prodige' »(p.125). On rappellera simplement que les clercs médiévaux ont sagement préféré à un peu euphonique Merdin un plus neutre Merlin.

30. Voir Joël H. Grisward, «Le Motif de l'épée jetée au lac : la mort d'Arthur et la mort de Batradtz », Romania, 90, 1969, pp. 289-340 et 473-514.

31. Voir Richard Trachsler, Clôtures du cycle arthurien. Étude et textes, Genève, Droz, «Publications romanes et françaises », CCXV, 1996.

32. Xavier de Langlais, Tristan hag Izold, éditions Al Liamm, 1958.

33. On n'en veut pour preuve - assez inénarrable - que l'annonce d'une «traduction française " du texte de Langlais par les responsables des éditions "Terre de brume " qui ne s'étaient même pas aperçus qu'ils ne proposaient ainsi qu'une caricature du texte de Bédier!

\section{AUTEUR}

\section{ALAIN CORBELLARI}

Université de Lausanne 